\title{
GENERALIZATIONS OF THE BERNOULLI POLYNOMIALS AND NUMBERS AND CORRESPONDING SUMMATION FORMULAS
}

TOMLINSON FORT

The Bernoulli polynomials and numbers have been generalized by Nörlund ${ }^{1}$ to the Bernoulli polynomials and numbers of higher order. The Bernoulli numbers have been generalized by Vandiver. Analogous polynomials and sets of numbers have been defined from time to time, witness the Euler polynomials and numbers and the so-called Bernoulli polynomials of the second kind. ${ }^{2}$

In the present paper a generalization is made which includes all the above and many other interesting classes of polynomials and corresponding sets of numbers. As a matter of fact the definition of new classes of polynomials by the processes of this paper is a simple matter. In this connection particular attention is called to $2,(\mathrm{~d}),(\mathrm{h}),(\mathrm{i})$, (j), (k), (1).

An important part of the paper is the development of a whole category of summation formulas related to the studied polynomials as the classical Euler-Maclaurin ${ }^{3}$ formula is related to the Bernoulli polynomials or as Taylor's formula is related to $(x-a)^{n}$.

It will be observed that the work could be varied in detail resulting in closely related polynomials and numbers to those which are obtained. The particular procedure adopted is chosen so as to generalize the Bernoulli polynomials and numbers as now usually defined. ${ }^{4}$

1. Definition of the polynomials and numbers. Let us be given two linear operators $P$ and $Q$ with their inverses $P^{-1}$ and $Q^{-1}$. We shall assume that $P$ reduces the degree of any polynomial by 1 and that $Q$ reduces the degree of any polynomial by $k \geqq 0$, that $P$ operating on a constant gives zero and that $Q$ operating on any polynomial of lesser degree than $k$ gives zero. We assume that $P, P^{-1}, Q, Q^{-1}$ each, where

Presented to the Society, December 29, 1941; received by the editors of the Transactions of this Society October 1, 1941; accepted by them, and later transferred to this Bulletin.

1 N. E. Nörlund, Differenzenrechnung, p. 119. For other generalizations see H. S. Vandiver, Proceedings of the National Academy of Sciences, vol. 23, p. 555. See also Leonard Carlitz, this Bulletin, abstract 47-7-296, also abstract 47-9-358.

${ }^{2}$ See C. Jordan, Calculus of Finite Differences, p. 265.

${ }^{3}$ For usage of the name Euler-Maclaurin see footnote of a paper by the author this Bulletin, vol. 45 (1939), p. 748. Usage in the present instance is that of Nörlund, loc. cit., p. 29.

4 See, for example, Nörlund, loc. cit., p. 17. 
applicable, gives a unique result except that it is permitted that the result of operating with $P^{-1}$ lack in uniqueness by an arbitrary additive constant and by $Q^{-1}$ by an additive arbitrary polynomial of degree less than $k$.

We assume, moreover, that we are given a set of polynomials $f_{n}(x)$ where $f_{n}(x)$ is of degree $n$, such that

$$
\begin{aligned}
f_{0}(x) & \equiv 1, \\
P f_{n}(x) & =n f_{n-1}(x),
\end{aligned}
$$

$$
\begin{aligned}
& Q^{-1} n(n-1) \cdots(n-k+1) f_{n-k}(x) \\
& \quad={ }_{n} L_{0} f_{n}(x)+n_{n} L_{1} f_{n-1}(x)+\frac{n(n-1)}{2}{ }_{n} L_{2} f_{n-2}(x)+\cdots \\
& \quad+C_{n, k} L_{n-k} f_{k}(x)+c_{1} x^{k-1}+c_{2} x^{k-2}+\cdots+c_{k}, 0 \leqq k \leqq n
\end{aligned}
$$

where ${ }_{n} L_{0}, \cdots,{ }_{n} L_{n-k}$ are independent of $x$ but uniquely determined. The $c$ 's may be determined constants or may be arbitrary depending upon the nature of the operator $Q^{-1}$.

From (3)

$$
\begin{aligned}
\frac{1}{n} & P Q^{-1} n(n-1) \cdots(n-k+1) f_{n-k}(x) \\
= & { }_{n} L_{0} f_{n-1}(x)+(n-1){ }_{n} L_{1} f_{n-2}(x)+\frac{(n-1)(n-2)}{2 !}{ }_{n} L_{2} f_{n-3}(x) \\
& \quad+\cdots+C_{n-1, k n} L_{n-k} f_{k-1}(x) \\
& \quad+[\text { terms of degree less than } k-1] .
\end{aligned}
$$

However,

$$
\begin{aligned}
\frac{1}{n} & Q^{-1} P n(n-1) \cdots(n-k+1) f_{n-k}(x) \\
= & Q^{-1}(n-1)(n-2) \cdots(n-k) f_{n-k-1}(x) \\
= & { }_{n-1} L_{0} f_{n-1}(x)+(n-1)_{n-1} L_{1} f_{n-2}(x) \\
& \quad+\frac{(n-1)(n-2)}{2 !}{ }_{n-1} L_{2} f_{n-3}(x)+\cdots+C_{n-1, k}+\cdots-1 L_{n-k} f_{k-1}(x) \\
& \quad+[\text { terms of degree less than } k] .
\end{aligned}
$$

We next assume

$$
P Q^{-1} f_{n-k}(x) \equiv Q^{-1} P f_{n-k}(x)+[\text { terms of degree less than } k] \text {. }
$$

Under this assumption 


$$
\begin{aligned}
& { }_{n} L_{0} f_{n-1}(x)+(n-1){ }_{n} L_{1} f_{n-2}(x)+\frac{(n-1)(n-2)}{2 !}{ }_{n} L_{2} f_{n-3}(x) \\
& \quad+\cdots+C_{n-1, k} L_{n-k} f_{k-1}(x) \\
& \quad \equiv{ }_{n-1} L_{0} f_{n-1}(x)+(n-1)_{n-1} L_{1} f_{n-2}(x) \\
& +\frac{(n-1)(n-2)}{2 !}{ }_{n-1} L_{2} f_{n-3}(x)+\cdots+C_{n-1, k n-1} L_{n-k} f_{k-1}(x) \\
& +[\text { terms of degree less than } k] .
\end{aligned}
$$

Equating coefficients we find

$$
{ }_{n} L_{0}={ }_{n-1} L_{0},{ }_{n} L_{1}={ }_{n-1} L_{1},{ }_{n} L_{2}={ }_{n-1} L_{2}, \cdots \text {. }
$$

In other words, the $L$ 's are independent of $n$. Henceforth we write simply $L_{0}, L_{1}, L_{2}, \cdots$. We let

$$
\begin{aligned}
F_{n}(x)= & L_{0} f_{n}(x)+n L_{1} f_{n-1}(x) \\
& +\frac{n(n-1)}{2 !} L_{2} f_{n-2}(x)+\cdots+L_{n} f_{0}(x), \quad n \geqq 0 .
\end{aligned}
$$

$$
Q F_{n}(x)=n(n-1) \cdots(n-k+1) f_{n-k}(x), \quad n \geqq k .
$$

For reasons of symmetry and consequent simplification of the sequel we do not take the $L_{n}$ 's just defined as our fundamental sequence of numbers but the set $g_{n}$ determined from the following equations $f_{n}(g)=L_{n}$ where subscripts are applied to $g$ rather than exponents in the expansion of $f_{n}(g)$. Such a determination is always possible and unique. We then write

$$
\begin{aligned}
F_{n}(x)= & f_{0}(g) f_{n}(x)+n f_{1}(g) f_{n-1}(x) \\
& +\frac{n(n-1)}{2 !} f_{2}(g) f_{n-2}(x)+\cdots+f_{n}(g) f_{0}(x), \quad n \geqq 0 .
\end{aligned}
$$

We choose to write this $f_{n}(x+g)$ of which more will be said later. We then have

$$
\begin{aligned}
F_{n}(x) & =f_{n}(x+g), \\
P F_{n}(x) & =n F_{n-1}(x), \quad n \geqq 1 .
\end{aligned}
$$

The polynomials $F_{n}(x)$ are the polynomials ${ }^{5}$ in which we are interested

${ }^{5}$ If we replace (2) by $P f_{n}(x)=n(n-1) \cdots(n-k+1) f_{n-h}(x)$ and add the requirement that the result of operating with $P$ on any polynomial of degree less than $h$ is zero, we are led to sequences of polynomials each of degree differing from that of the previous by $h$, a somewhat more general situation than that treated in the text. 
and the numbers $g_{n}$ constitute the corresponding sequence of numbers.

2. Special cases. ${ }^{6}$ We now consider these special cases.

(a) Bernoulli polynomials and numbers:

$$
P=\frac{d}{d x}, \quad Q=\Delta, \quad f_{n}(x)=x^{n} .
$$

(b) Bernoulli polynomials and numbers of the second kind:

$$
P={ }_{\omega}, \quad Q=\frac{d}{d x}, \quad f_{n}(x)=x^{(n)}=x(x-\omega) \cdots(x-(n-1) \omega) .
$$

(c) Bernoulli polynomials and numbers of higher order as defined by Nörlund:

$$
P=\frac{d}{d x}, \quad Q={ }_{\omega_{1} \cdot \omega_{\omega_{k}}}^{\Delta k}, \quad f_{n}(x)=x^{n}, \quad k>1 .
$$

(d) Bernoulli polynomials and numbers of the second kind of higher order : ${ }^{7}$

$$
P=\Delta_{\omega}^{\Delta}, \quad Q=\frac{d^{k}}{d x^{k}}, \quad f_{n}(x)=x^{(n)}, \quad k>1 .
$$

(e) Bernoulli polynomials with Bernoulli numbers of higher order as defined by Vandiver:

$$
P=\frac{d}{d x}, \quad Q={ }_{\omega_{1}} \Delta^{k}{ }^{k}, \omega_{k}, \quad f_{n}(x)=(x+l)^{n} .
$$

Vandiver's numbers are the $L_{n}$ 's of the text rather than the $g_{n}$ 's. (f) Euler polynomials:

$$
P=\frac{d}{d x}, \quad Q=M, \quad f(x)=x^{n} .
$$

${ }^{6}$ Certain notation in this section is as follows:

$$
\begin{array}{rlrl}
\Delta f(x) & =f(x+1)-f(x) ; & & \Delta f(x)=\frac{1}{\omega}(f(x+\omega)-f(x)) ; \\
\Delta \quad f(x) & =\Delta \Delta \cdots \Delta f(x) ; & & \Delta f(x)=\frac{1}{2}(f(x+\omega)+f(x)) ; \\
\omega_{1}, \cdots, \omega_{1} & \omega_{\omega_{k}} \\
T f(x) & \stackrel{f}{=} \frac{(q x)-f(x)}{q x-x}, & &
\end{array}
$$

7 This name is applied through analogy with the Bernoulli polynomials of higher order as defined by Nörlund and as a natural extension of the Bernoulli polynomials of the second kind already referred to. 
(g) Euler polynomials of higher order:

$$
P=\frac{d}{d x}, \quad Q=M^{k}, \quad f(x)=x^{n} .
$$

$(\mathrm{h})^{8}$

$$
P=\frac{d}{d x}, \quad Q=T^{k}, \quad f(x)=x^{(n)}, \quad k>0
$$

$$
P=\frac{d}{d x}, \quad Q={ }_{\omega_{1},{ }^{k} \cdot \omega_{k}},
$$

$f_{n}(x)$ any set of Appell ${ }^{9}$ polynomials.

$$
P=\Delta, \quad Q=\frac{d^{k}}{d x^{k}}
$$

$f_{n}(x)$ any set of Appell polynomials of the second kind.

$$
P=a_{0} D^{r}+a_{1} D^{r-1}+\cdots+a_{r-1} D \equiv R(D),
$$

where $D=d / d x$ and $a_{0}, a_{1}, \cdots, a_{r-1}$ are constants; $a_{r-1} \neq 0, Q=b_{0} \Delta^{r}$ $+b_{1} \Delta^{r-1}+\cdots+b_{s}, b_{0}, b_{1}, \cdots, b_{s}$ being constants. $f_{n}(x)$ are polynomials obtained by successive solutions of the equation

$$
R(D) f_{n}(x)=n f_{n-1}(x) .
$$

(1) Interchange $D$ and $\Delta$ in $(\mathrm{j})$.

3. Summation formulas. In the remainder of this paper we shall assume $f_{n}(0)=0$.

We first prove a lemma with reference to $f_{n}(x)$. We shall prove that

$$
\begin{aligned}
f_{n}(x+h)= & f_{n}(x)+n f_{n-1}(x) f_{1}(h) \\
& +\frac{n(n-1)}{2 !} f_{n-2}(x) f_{2}(h)+\cdots+f_{n}(h) .
\end{aligned}
$$

To do this we write

$$
f_{n}(x+h)=b_{0}(x)+b_{1}(x) f_{1}(h)+b_{2}(x) f_{2}(h)+\cdots+b_{n}(x) f_{n}(h),
$$

where the b's are as yet undetermined. Such an expression is possible

${ }^{8}$ F. H. Jackson, Quarterly Journal of Mathematics, vol. 41 (1909-1910), p. 195.

${ }^{9}$ We understand by a set of Appell polynomials a set of polynomials such that $(d / d x) f_{n}(x)=n f_{n-1}(x)$ and of the second kind such that

$$
\underset{\omega}{\Delta} f_{n}(x)=n f_{n-1}(x) .
$$


since $f_{n}(x+h)$ is a polynomial of the $n$th degree in $h$. We determine the $b$ 's by successively applying the operator $P$ to (13) as a function of $h$ and then letting $h=0$ remembering that $f_{n}(0)=0$.

We have attached a meaning to $f_{n}(x+g)$. With this meaning in mind we prove that

$$
f_{n}((x+h)+g)=f_{n}(x+(h+g)) .
$$

Exponents are applied to $x$ and $h$, subscripts to $g$. Expansion of the left-hand member of (15) is the same as that given in (9) with $(x+h)$ replacing $x$. The whole expansion carried out on both sides of (12) is exactly the same as the expansions of $((x+h)+g)^{n}$ and $(x+(h+g))^{n}$ with the exponents replaced by the $f$-function. Thus $f_{k}(x)$ replaces $x^{k}$. Inasmuch as the binomial expansion yields an identity so does the expansion in terms of $f$ that we are considering.

Now consider any polynomial of degree $m+k$ which we write

$$
\begin{aligned}
\psi(g) & =a_{0}+a_{1} f_{1}(x)+\cdots+a_{m+k} f_{m+k}(x), \\
\psi(x+g) & =a_{0}+a_{1} f_{1}(x+g)+\cdots+a_{m+k} f_{m+k}(x+g) \\
& \equiv a_{0}+a_{1} F_{1}(x)+\cdots+a_{m+k} F_{m+k}(x) .
\end{aligned}
$$

Hence by (8) $Q \psi(x+g)=a_{k}(k !)+a_{k+1}(k+1) \cdots 2 f_{1}(x)+\cdots$ $+a_{m+k}(m+k)(m+k-1) \cdots(m+1) f_{m}(x)$. That is, $Q \psi(x+g)=P^{k} \psi(x)$, $m+k \geqq 0$. Similarly

$$
Q \psi(x+h+g)=P^{k} \psi(x+h) .
$$

We apply Taylor's formula symbolically to this, that is, we write

$$
Q \psi(x+h+g)=c_{0}(x)+c_{1}(x) F_{1}(h)+\cdots+c_{m}(x) F_{m}(h)
$$

and determine the $c$ 's by first letting $h=-g$ and then successively applying $P$, remembering (10) and letting $h=-g$ after each application. ${ }^{10}$ We get

Hence by (15)

$$
Q \psi(x+h+g)=Q \psi(x)+\sum_{v=1}^{m} \frac{F_{v}(h)}{v !} P^{v} Q \psi(x) .
$$

$$
P^{k} \psi(x+h)=Q \psi(x)+\sum_{v=1}^{m} \frac{F_{v}(h)}{v !} P^{v} Q \psi(x) .
$$

This is a polynomial identity. Let $\psi(x)=P^{-k} \chi(x)$ where some particular determination is chosen in case $P^{-k}$ is not unique. Then

$$
\chi(x+h)=\sum_{v=0}^{m} \frac{F_{v}(h)}{v !} P^{v} Q P^{-k} \chi(x) .
$$

${ }^{10}$ Here, of course, when $h$ is replaced by $g$ an exponent is changed to a subscript. 
Like (16) this is a polynomial identity. It includes as special cases Taylor's formula, the Euler-Maclaurin formula ${ }^{11}$ of classical mathematics, the Boole formula, and so on.

Formula (17) will be written out in detail for two interesting special cases.

Let

$$
\begin{aligned}
& P=\frac{d}{d x}, \quad Q={ }_{\omega}{ }^{k}, \quad P^{-1}=\int_{0}^{x} d x, \quad k=1,2, \cdots ; \\
& \chi(x+h)=\Delta_{\omega}^{k} \int_{0}^{x} \int_{0}^{x_{1}} \cdots \int_{0}^{x_{k-1}} \chi\left(x_{k}\right) d x_{1} d x_{2} \cdots d x_{k} \\
& +B_{1}^{(k)}(h) \underset{\omega}{\Delta^{k}} \int_{0}^{x} \int_{0}^{x_{1}} \cdots \int_{0}^{x_{k-1}} \chi\left(x_{k-1}\right) d x_{1} d x_{2} \cdots d x_{k-1} \\
& +\cdots+\frac{1}{(k-1) !} B_{k-1}^{(k)}(h) \Delta_{\omega}^{k} \int_{0}^{x} \chi\left(x_{1}\right) d x_{1} \\
& +\frac{1}{k !} B_{k}^{(k)}(h) \Delta_{\omega}^{k} \chi(x)+\cdots+\frac{1}{m !} B_{m}^{(k)}(h) \Delta_{\omega}^{k} \frac{d^{m-k}}{d x^{m-k}} \chi(x) .
\end{aligned}
$$

Secondly we let $\left.\left.P=\Delta, Q=d^{k} / d x^{k}, P^{-1}=\sum_{0}^{x-1} \equiv \sum\right]_{x}-\sum\right]_{0}$ where $\sum=\Delta^{-1}$

$$
\begin{aligned}
\chi(x+h)= & \frac{d^{k}}{d x^{k}} \sum_{x_{1}=0}^{x-1} \sum_{x_{2}=0}^{x_{1}-1} \cdots \sum_{x_{k}=0}^{x_{k-1}-1} \chi\left(x_{k}\right) \\
& +b_{1}^{(k)}(h) \frac{d^{k}}{d x^{k}} \sum_{x_{1}=0}^{x-1} \sum_{x_{2}=0}^{x_{1}-1} \cdots \sum_{x_{k-1}=0}^{x_{k-2}-1} \chi\left(x_{k-1}\right)+\cdots \\
& +\frac{1}{(k-1) !} b_{k-1}^{(k)}(h) \frac{d^{k}}{d x^{k}} \sum_{0}^{x-1} \chi\left(x_{1}\right) \\
& +\frac{1}{k !} b_{k}^{(k)}(h) \frac{d^{k}}{d x^{k}} \chi(x)+\cdots \\
& +\frac{1}{m !} b_{m}^{(k)}(h) \frac{d^{k}}{d x^{k}} \Delta^{m-k} \chi(x) .
\end{aligned}
$$

In the above formulas $B_{j}^{(k)}(h)$ is the Bernoulli polynomial of order $k$ and degree $j$, similarly $b_{j}^{(k)}(h)$ is the analogous polynomial which we designate as the Bernoulli polynomial of the second kind of order $k$ and degree $j$.

11 Nörlund, p. 29. 
Formula (18) should be compared with a related formula of Nörlund. ${ }^{12}$ If the operator $\sum_{x_{1}=0}^{x-1} \sum_{x_{2}=0}^{x_{1}-1} \cdots \sum_{x_{k}=0}^{x_{k-1}-1}$ is applied to both sides of (18) we get an interesting result which should be compared with a formula studied by the author in an earlier paper. ${ }^{13}$

4. A remainder formula. Let us assume that $\psi(x)$ is no longer a polynomial but that it is such a function that all operations applied to it in the sequel are meaningful. We consider formula (16) as our fundamental form.

We assume that $Q \psi(x)+\sum_{v=0}^{m}\left(F_{v}(t) / v !\right) P^{v} Q \psi(x)$ involves $m+1$ points, dependent upon $x$ alone, which we call set 1 . Some or all of these may coincide. For example, $\Delta^{m} Q \psi(x)$ involves $m+1$ distinct points and $\left(d^{m} / d x^{m}\right) Q \psi(x) m+1$ coincident points. Let $x$ be a constant and $t$ a variable. When $t$ is such that $x+t$ coincides with any one of the points of set 1

$$
P^{k} \psi(x+t)-\left[Q \psi(x)+\sum_{v=1}^{m} \frac{F_{v}(t)}{v !} P^{v} Q \psi(x)\right]
$$

vanishes, inasmuch as every term is identical with the like term which is built for the polynomial of degree $m$ which coincides with $\psi(x)$ at the points of set 1 . It has been remarked that (16) is an identity for a polynomial. Now denote by $p(t)$ a polynomial of degree $m+1$ which vanishes when $x+t$ coincides with any one of the $m+1$ points of set 1 . Let the coefficient of $t^{m+1}$ in $p(t)$ be 1 . Choose a constant $T$ such that

$$
P^{k} \psi(x+t)-\left[Q \psi(x)+\sum_{v=1}^{m} \frac{F_{v}(t)}{v !} P^{v} Q \psi(x)\right]-T p(t)
$$

vanishes at the additional point where $t=h$. Then by repeated application of Rolle's theorem $(m+1) ! T=d^{m+1}\left(P^{k} \psi(\xi)\right) / d x^{m+1}$ where $\xi$ is somewhere between the extreme values of the $(m+2)$ points composed of the points of set 1 and the additional point $x+h$. We have a final form for the remainder to formula (16),

$$
R=\frac{1}{(m+1) !} p(h) \frac{d^{m+1}}{d x^{m+1}}\left(P^{k} \psi(\xi)\right) .
$$

The corresponding formula for (17) is

$$
R=\frac{1}{(m+1) !} p(h) \frac{d^{m+1}}{d x^{m+1}} \chi(\xi)
$$

LEHIGH UNIVERSITY

${ }^{12}$ Loc. cit., p. 160.

${ }^{13}$ Loc. cit. 